\title{
Analysis of the Striato-Thalamo-Cortical Connectivity on the Cortical Surface to Infer Biomarkers of Huntington's Disease ${ }^{\star}$
}

\author{
Linda Marrakchi-Kacem ${ }^{1,2}$, Christine Delmaire ${ }^{2,4}$, Alan Tucholka ${ }^{1,2,3}$, \\ Pauline Roca ${ }^{1,2}$, Pamela Guevara ${ }^{1,2}$, Fabrice Poupon ${ }^{1,2}$, Jérôme Yelnik ${ }^{5}$, \\ Alexandra Durr ${ }^{5}$, Jean-François Mangin ${ }^{1,2}$, \\ Stéphane Lehericy ${ }^{2,4}$, and Cyril Poupon ${ }^{1,2}$ \\ 1 Neurospin, CEA, Gif-sur-Yvette, France \\ 2 Institut Fédératif de Recherche 49, Gif-sur-Yvette, France \\ 3 Parietal Team, INRIA Saclay-Ile-de-France, Saclay, France \\ ${ }^{4}$ CENIR, Pitié Salpêtriére Hospital, Paris, France \\ 5 CRICM, Inserm/UPMC, UMR-S 975, Paris, France
}

\begin{abstract}
The deep brain nuclei play an important role in many brain functions and particularly motor control. Damage to these structures result in movement disorders such as in Parkinson's disease or Huntington's disease, or behavioural disorders such as Tourette syndrome. In this paper, we propose to study the connectivity profile of the deep nuclei to the motor, associative or limbic areas and we introduce a novel tool to build a probabilistic atlas of these connections to the cortex directly on the surface of the cortical mantel, as it corresponds to the space of functional interest. The tool is then applied on two populations of healthy volunteers and patients suffering from severe Huntington's disease to produce two surface atlases of the connectivity of the basal ganglia to the cortical areas. Finally, robust statistics are used to characterize the differences of that connectivity between the two populations, providing new connectivity-based biomarkers of the pathology.
\end{abstract}

Keywords: deep nuclei, diffusion, tractography, Q-Ball imaging, connectivity, surface atlases.

\section{Introduction}

The deep brain nuclei include the basal ganglia and the thalamus. The basal ganglia include the caudate nuclei and the putamen, which constitute the striatum, and the globus pallidus. The striatum receives afferents from the cerebral cortex. Most areas of the neocortex except the primary visual and auditive areas have projections on to the striatum. The striatum projects to the external and

\footnotetext{
* This work was funded by the Association France Parkinson, the Ecole des Neurosciences Paris-Ile-de-France, the ANR MNP, the DHOS AP-HP INSERM and the CHDI High Q Foundation.
} 
internal segments of the globus pallidus and the internal globus pallidus projects in turn to the thalamus. In Huntington's disease, these pathways are disrupted. Imaging can provide biomarkers that may improve the understanding of the pathophysiology of the disease. Diffusion-weighted (DW) magnetic resonance imaging (dMRI) is now a well established technique to infer the anatomical connectivity in vivo. dMRI can probe the anisotropy of the displacement of water molecules at microscopic scales in tissues, revealing their structural organization. In the case of the brain white matter (WM), the more recent local mathematical models of the diffusion process proposed in the literature clearly depict some displacement $(\mathrm{PDF})$ or orientational probability distribution (ODF), the maxima of which corresponds to the direction of the underlying axonal fibers. Tractography algorithms were developed to more or less robustly recover the global connectome using this local information and are now widely used to perform studies of the anatomical connectivity and of its disorders.

Several studies have been conducted about the striato-pallido-thalamo-cortical connectivity 1223 . The first preclinical studies have focused on the functional role played by the thalamus receiving afferents from the cortex and sending projections back to it. Then, surprisingly, most of the clinical studies focused on parcelating the functional territories of the deep structures having the a priori knowledge of their connectivity to the cortical mantel and a labeling of the cortex into functional areas 45], whereas none of them has tried to infer the connectivity profile of the deep nuclei (and possibly their sub-territories) onto the cortical mantel. We believe that this approach may be of interest as the deep nuclei are connected to most of the cortical regions. As shown previously, many motor disorders may be linked to putative disruption of the neuronal pathways between specific nuclei and cortical areas. Therefore, building statistical atlases of the connectivity of these nuclei to the cortical mantel for different populations (healthy volunteers versus patients) may help understanding which cortical areas are significantly "disconnected" for each deep structure from both a qualitative and quantitative point of view.

In this paper we propose a novel tool to study the striato-thalamo-cortical connectivity at the surface of the cortical mantel, dedicated to group studies. This tool relies on the construction of surface probabilistic atlas that can be used to quantify the probability of connection of a given nucleus to predefined cortical regions of interest for a given subject or for a given population. We will show that the measure of the probabilities provided by this tool can be used to detect and characterize the axonal disruptions occurring in Huntington's disease. The paper is organized as follows: after introducing the methods involving several image processing steps to project the information onto the cortical surface, the technique is applied to two populations of controls and patients; robust statistics are then performed to detect the functional areas of the cortex that were significantly atrophiated. We show that this tool brings complementary information to the previous approaches that have focused on the study of the Huntington's disease (HD) using dMRI 6 67 ]. 


\section{Methods}

The method is based on the construction of probabilistic atlases at the surface of the cortical mantel of the connections between the deep nuclei and the cortical areas. This task relies on the use of both $T_{1}$-weighted data to extract the deep structures and the cortical mantel and on high angular resolution diffusionweighted data (HARDI) to recover the anatomical connectivity. This section is devoted to the description of the different steps of image processing required to build the atlases and to analyze them using adequate statistics.

\subsection{Structural Database}

A database of 17 healthy volunteers and 17 HD patients was acquired on a Tim Trio 3T MRI system (Siemens, Erlangen) in the frame of a clinical project dedicated to the study of Huntington's disease (HD, HDTrack project). All subjects signed an informed consent and the imaging protocol was approved by the Local Ethical Committee. $T_{1}$-weighted and DW data were acquired using the following sequence parameters: $3 D M P R A G E \mathrm{TE} / \mathrm{TR}=2.98 \mathrm{~ms} / 2.3 \mathrm{~s}, \mathrm{FOV}=256 \mathrm{~mm}$, matrix $256 \times 256, \mathrm{TH}=1.1 \mathrm{~mm}, 160$ slices per slab, RBW=240Hz/pixel; Singleshot twice refocused spin-echo $D W$-EPI $\mathrm{TE} / \mathrm{TR}=86 \mathrm{~ms} / 12 \mathrm{~s}, \mathrm{FOV}=256 \mathrm{~mm}$, matrix $128 \times 128, \mathrm{TH}=2 \mathrm{~mm}, 80$ slices, acceleration factor 2, partial Fourier $6 / 8, \mathrm{RBW}=1630 \mathrm{~Hz} /$ pixel, $\mathrm{b}=1000 \mathrm{~s} / \mathrm{mm}^{2}, 50$ diffusion directions uniformly distributed. EPI distortions caused by susceptibility effects were corrected with BrainVISA and using a further phase map acquisition to evaluate the distortions along the phase axis. Each DW data was matched to the corresponding $T_{1}$-weighted data using a rigid $3 \mathrm{D}$ transform.

\subsection{Segmentation of the Deep Nuclei and the Cortex}

The deep nuclei and cortex were segmented from the $T_{1}$-weighted data. For each subject, eight deep structures were automatically delineated using the method described in [9]: left caudate (LCd), left putamen (LPu), left thalamus (LTh), left globus pallidus (LGP), right caudate $(\mathrm{RCd})$, right putamen $(\mathrm{RPu})$, right globus pallidus (RGP), right thalamus (RTh). The automatic segmentations were then checked by a neuro-anatomist and corrected manually if needed.

FreeSurfer was used to extract the interface between the white matter (WM) and the cortex for all the subjects. The vertices of the obtained surface meshes are in direct correspondence [10]. This property is mandatory to conveniently match the different subjects [1]. In order to synthetize the information stemming from different subjects, an average surface was computed for the two populations from the individual interfaces extracted for all the subjects.

\subsection{Inference of the Striato-Pallido-Thalamo-Cortical Connectivity}

Tractography. In order to infer the connectivity between the deep nuclei and the cortical surface, a streamline probabilistic tractography algorithm was 
employed as described in 12. This choice was motivated by efficacy of such algorithms to deal with complex fiber geometries (crossings, fannings) compared to streamline deterministic algorithms. Bayesian or global algorithms could have been chosen, but at the price of much longer computation times. A robust mask of the brain white matter was built from the $T_{1}$-weighted data and 10 probabilistic streamlines were processed for each voxel of the mask. An analytical Q-ball model described in [13] was used to estimate the local underlying orientation distribution function (ODF) using a spherical harmonics order 6 and a regularization factor equal to 0.006 . At each step of the streamlining, the most likely direction is determined from the ODF and a random direction is chosen in a cone of aperture $30^{\circ}$ around the optimal direction. This whole brain tractography leads to individual tractograms containing $10^{6}$ fibers on average.

The intersection of each fiber with the deep nuclei was computed. Starting from one of the two extremities of a fiber, if $n$ is the first nucleus to be met by the points of the fiber, the portion of the fiber linking the chosen fiber extremity to $n$ is attributed to $n$. This process provides the fibers crossing each nucleus.

Striato-Pallido-Thalamo-Cortical Connectivity Matrix. A connectivity matrix was computed to evaluate at each vertex of the cortical surface the number of fibers linking it to each nucleus. For each nucleus $n$, the number of fibers connecting it to each region of the cortex is obtained by computing the intersection between the fibers crossing $n$ and the WM/cortex interface. The values related to each nucleus $n$ are stored in a line of a sparse matrix $C_{s}^{r a w}$ for subject $s$. This matrix is then smoothed over the surface to account for a reasonable uncertainty on the tracking result giving a matrix $C_{s}$ for the subject $s$ [14].

\subsection{Probabilistic Surface Atlases of the Connectivity}

The goal is now to merge all the striato-thalamo-pallido-cortical connectivity information stemming from all the subjects into a single probabilistic atlas. This task must be repeated for the two different populations.

For any subject $s$, its connectivity matrix contains for each nucleus $n$ and for any vertex position $v$ an approximation $C_{s}(n, v)$ of the number of fibers connecting $n$ to $v$. The probability $p_{s}(n, v)$ of connection between $n$ and $v$ is the ratio between $C_{s}(n, v)$ and the global number of fibers coming from all the nuclei that project onto the cortical mantel of the subject $s$. Let $\mathcal{N}$ be the set of nuclei and $\mathcal{V}_{s}$ be the set of positions of the vertices belonging to the $\mathrm{WM} /$ cortex interface for the subject $s$. For any $n \in \mathcal{N}$ and any $v \in \mathcal{V}_{s}$,

$$
p_{s}(n, v)=\frac{C_{s}(n, v)}{\sum_{n^{\prime} \in \mathcal{N}} \sum_{v^{\prime} \in \mathcal{V}_{s}} C_{s}\left(n^{\prime}, v^{\prime}\right)}
$$

For a given nucleus $n$, the set of values $p_{s}(n, v)$ associated to the vertices belonging to the $\mathrm{WM} /$ cortex interface represents the probabilistic atlas of the connectivity of this nucleus $n$ for the subject $s$. For a given population $\mathcal{P}$, averaging the connectivity matrices $C_{s}$ provides the wanted probabilistic atlas for all the 
nuclei $n$, since vertices of the individual surfaces provided by FreeSurfer are in direct correspondence. $\left(\forall s, \forall s^{\prime}, \mathcal{V}_{s}=\mathcal{V}_{s^{\prime}}=\mathcal{V}\right)$.

$$
C_{\mathcal{P}}(n, v)=\frac{\sum_{s \in \mathcal{P}} C_{s}(n, v)}{\operatorname{card}(\mathcal{P})}
$$

The probability $p_{\mathcal{P}}(n, v)$ of connection of $n \in \mathcal{N}$ to $v \in \mathcal{V}$ is the ratio between $C_{\mathcal{P}}(n, v)$ and the mean total number of fibers coming from all the nuclei that project onto the average cortical mantel for the population $\mathcal{P}$. For a given nucleus $n$, the set of values $p_{\mathcal{P}}(n, v)$ computed over all the vertices belonging to the WM/cortex average interface of $\mathcal{P}$ represents the probabilistic atlas of the connectivity of $n$, for the population $\mathcal{P}$.

$$
p_{\mathcal{P}}(n, v)=\frac{C_{\mathcal{P}}(n, v)}{\sum_{n^{\prime} \in \mathcal{N}} \sum_{v^{\prime} \in \mathcal{V}} C_{\mathcal{P}}\left(n^{\prime}, v^{\prime}\right)}
$$

Probabilistic atlases have been represented for each nucleus using a gradient color ranging from red corresponding to a high probability of connection to gray corresponding to a low probability of connection. Thus, simple qualitative comparisons can already be performed between the two populations of healthy subjects and patients.

\subsection{Atlas Differences between Populations}

The HDTrack database includes 2 populations: healthy subjects $(\mathrm{H})$ and subjects suffering from severe Huntington disease (HD). Probabilistic connectivity atlases computed, for each nucleus, on each subject of each population can be used to perform some statistical comparisons between the two populations.

A Mann Whitney test was used to compare the probability of connection of each nucleus to the cortex between the two populations. This choice was motivated by the lack of assumption about the normality of the data.

For a given population $\mathcal{P}$, the probability $p_{s}(n, v)$ was considered as a random variable $X_{n, v}^{\mathcal{P}}$ taking different values for the different subjects $s$. Using the MannWhitney test on the two random variables $X_{n, v}^{\mathcal{P}_{\mathcal{H}}}$ and $X_{n, v}^{\mathcal{P}_{\mathcal{H}} \mathcal{D}}$ corresponding to the two populations of controls and patients, we detected trends of significant difference $(p$-value $<0.05)$ of the connectivity, for the given nucleus $n$ and the given vertex position $v$, between the two populations $\mathcal{P}_{\mathcal{H}}$ and $\mathcal{P}_{\mathcal{H} \mathcal{D}}$.

\section{Results and Discussion}

Probabilistic Connectivity Atlases. A probabilistic connectivity atlas was computed for the two populations (H) and (HD), and for all the nuclei, as depicted in figure 1. The left and right columns correspond to the left and right structures respectively. The intensity of the red color is proportional to the level of connectivity. We can observe that most of the connections of a given nucleus are located in the ipsilateral hemisphere. The globus pallidus does not present 


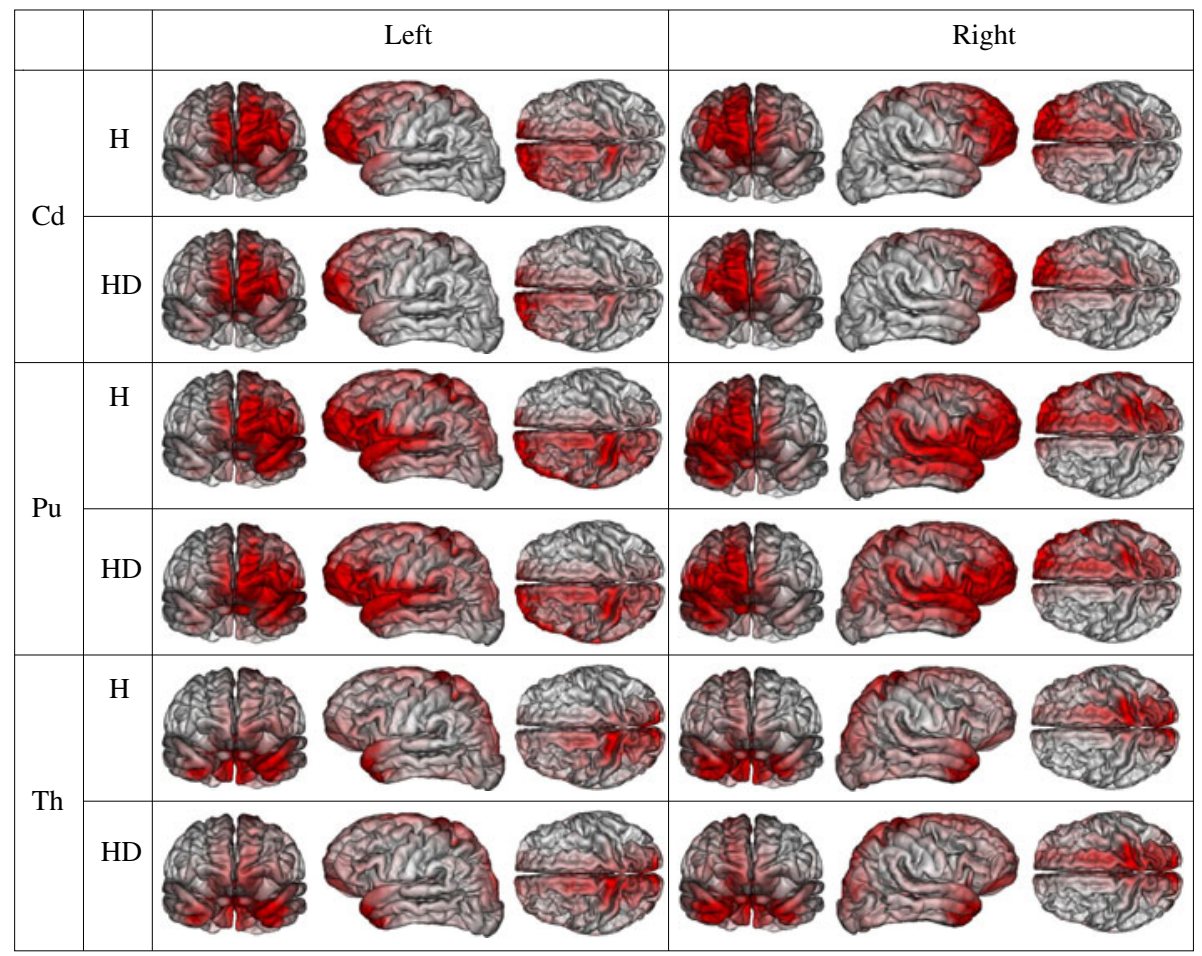

Fig. 1. Surface probabilistic striato-thalamo-cortical connectivity atlases computed for each population: healthy subjects and patients suffering from Huntington's disease

any projection to the cortex and is consequently not shown in the figure. As expected, the caudate nuclei project mostly to the frontal areas and to the premotor and motor areas. The putamen is mainly connected to the motor and premotor areas, to the frontal ventro-lateral areas, and to the temporal superior area. The thalami have projections to the entire cortex.

Statistical Comparison Test. For each nucleus, the Mann Whitney test allowed the detection of the vertices presenting a significant connectivity difference between the two populations. The regions corresponding to these vertices were represented using a color palette as shown in figure 2 ,

Reduction of the connections of the caudate nucleus was greater than the reduction of the connections of the putamen and thalami, especially in the Broca's area, the motor area, and the temporal superior area. The connectivity differences observed obtained by the comparison of the probabilistic connectivity atlases were in good agreement with the symptoms observed on the HD patients. As an example, the probability of connection of the left caudate, left putamen, 
Gradient palette for statistical test $\mathrm{p}$ values

0 0.05

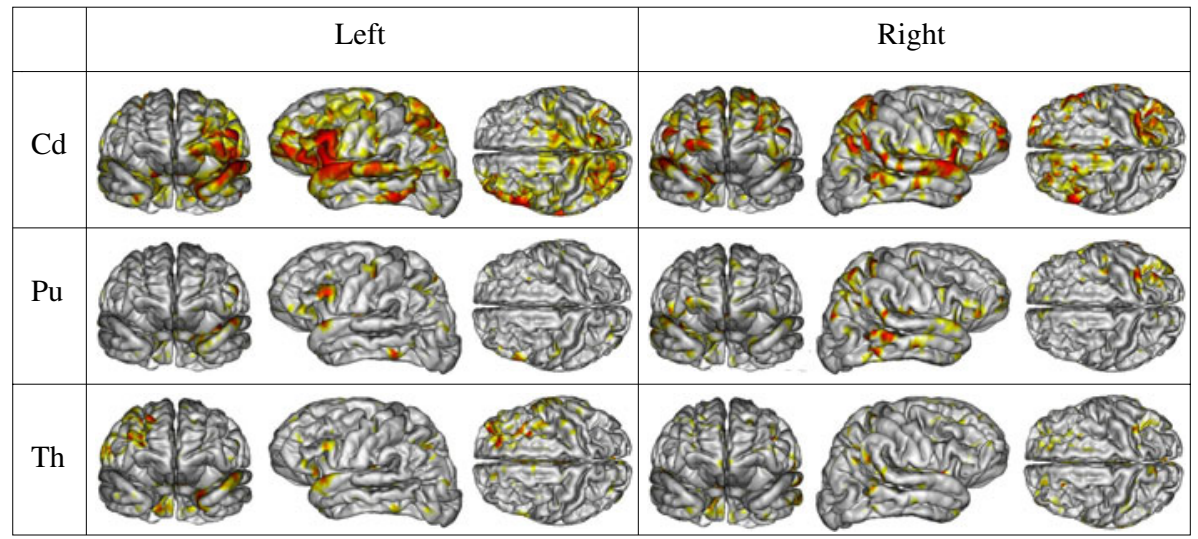

Fig. 2. Cortical regions presenting a significant connectivity difference between healthy subjects and Huntington's patients per nucleus stemming from statistical analysis of their striato-thalamo-cortical surface atlases

left thalamus and right caudate to the Broca's area was significantly different between the two populations. The obtained statistics clearly indicate that the caudate nucleus has the most atophiated connectivity and this atrophy is more predominant in the left caudate nucleus.

\subsection{Conclusion}

In this paper, we introduced a novel tool for the study of the striato-thalamocortical connectivity relying on surface probabilistic connectivity atlases. Projecting the connectivity profile onto the cortical mantel is relevant as it enables to detect disruption of these connections directly in the frame of functional areas. We used this novel tool on a population of healthy subject and on a population of patients suffering of the Huntington's disease and we proved that it was adequate to detect differences of the striato-thalamo-cortical connectivity between the two populations, using robust statistics. The structural lesions that were detected were in good agreement with the known physiopathology of Huntington's disease. In the future, this tool will be used to investigate several neurodegenerative pathologies involving the basal ganglia, and improvements will be done to provide accurate information about areas with atrophy, such as performing longitudinal studies of the pathology.

\section{References}

1. Alexander, G.E., DeLong, M.R., Strick, P.L.: Parallel organization of functionally segregated circuits linking basal ganglia and cortex. Annual Review of Neuroscience 9(1), 357-381 (1986) 
2. Barbas, H., Pandya, D.: Architecture and frontal cortical connections of the premotor cortex (area 6) in the rhesus monkey. The Journal of Comparative Neurology 256, 211-228 (1987)

3. Alexander, G.E., Crutcher, M.D.: Functional architecture of basal ganglia circuits: neural substrates of parallel processing. Trends in Neurosciences 13(7), 266-271 (1990)

4. Behrens, T., Johansen-Berg, H., Woolrich, M.W., Smith, S.M., Wheeler-Kingshott, C.A.M., Boulby, P.A., Barker, G.J., Sillery, E.L., Sheehan, K., Ciccarelli, O., Thompson, A.J., Brady, J.M., Matthews, P.M.: Non-invasive mapping of connections between human thalamus and cortex using diffusion imaging. Neuroscience 6 , 750-757 (2003)

5. Draganski, B., Kherif, G., Kloppel, S., Cook, P.A., Alexander, D.C., Parker, G.J., Deichmann, R., Ashburner, J., Frackowiak, R.S.: Evidence for segregated and integrative connectivity patterns in the human basal ganglia. The Journal of Neuroscience 28, 7143-7152 (2008)

6. Bohanna, I., Georgiou-Karistianis, N., Hannan, A.J., Egan, G.F.: Magnetic resonance imaging as an approach towards identifying neuropathological biomarkers for huntington's disease. Brain Res. Rev. 58(1), 209-225 (2008)

7. Douaud, G., Behrens, T.E., Poupon, C., Cointepas, Y., Jbabdi, S., Gaura, V., Golestani, N., Krystkowiak, P., Verny, C., Damier, P., Bachoud-Levi, A.C., Hantraye, P., Remy, P.: In vivo evidence for the selective subcortical degeneration in huntington's disease. Neuroimage 46, 958-966 (2009)

8. Rosas, H.D., Lee, S.Y., Bender, A.C., Zaleta, A.K., Vangel, M., Yu, P., Fischl, B., Pappu, V., Onorato, C., Cha, J.H., Salat, D.H., Hersch, S.M.: Altered white matter microstructure in the corpus callosum in huntington's disease: Implications for cortical disconnection. NeuroImage 49(4), 2995-3004 (2010)

9. Marrakchi-Kacem, L., Poupon, C., Mangin, J.F., Poupon, F.: Multi-contrast deep nuclei segmentation using a probabilistic atlas. In: ISBI (2010)

10. Argall, B.D., Saad, Z.S., Beauchamp, M.S.: Simplified intersubject averaging on the cortical surface using suma. Human Brain Mapping 27, 14-27 (2006)

11. Fischl, B., Sereno, M.I., Tootell, R.B., Dale, A.M.: High-resolution intersubject averaging and a coordinate system for the cortical surface. Human Brain Mapping 8, 272-284 (1999)

12. Perrin, M., Poupon, C., Cointepas, Y., Rieul, B., Golestani, N., Pallier, C., Riviere, D., Constantinesco, A., LeBihan, D., Mangin, J.F.: Fiber tracking in q-ball fields using regularized particle trajectories. In: Christensen, G.E., Sonka, M. (eds.) IPMI 2005. LNCS, vol. 3565, pp. 52-63. Springer, Heidelberg (2005)

13. Descoteaux, M., Angelino, E., Fitzgibbons, S., Deriche, R.: Regularized, fast, and robust analytical q-ball imaging. Magnetic Resonance in Medicine 58(3), 497-510 (2007)

14. Roca, P., Riviere, D., Guevara, P., Poupon, C., Mangin, J.F.: Tractography-based parcellation of the cortex using a spatially-informed dimension reduction of the connectivity matrix. In: Yang, G.-Z., Hawkes, D., Rueckert, D., Noble, A., Taylor, C. (eds.) MICCAI 2009. LNCS, vol. 5761, pp. 935-942. Springer, Heidelberg (2009) 